\title{
Structure dependent negative magnetoresistance of amorphous carbon thin films
}

\author{
Awais Siddique Saleemi $^{\mathrm{a}, \mathrm{b}}$, Rajan Singh ${ }^{\mathrm{a}, \mathrm{b}}$, Zhaochu Luo ${ }^{\mathrm{a}, \mathrm{b}}$, Xiaozhong Zhang ${ }^{\mathrm{a}, \mathrm{b} *}$ \\ ${ }^{a}$ Key Laboratory of Advanced Materials (MOE), School of Materials Science and \\ Engineering, Tsinghua University, Beijing 100084, People's Republic of China \\ ${ }^{\mathrm{b}}$ Beijing National Center for Electron Microscopy, Beijing 100084, People's Republic \\ of China \\ * Corresponding Author. E-mail: xzzhang@tsinghua.edu.cn
}

\begin{abstract}
Negative magnetoresistance (MR) up to $13 \%$ was observed at $2 \mathrm{~K}$ under the magnetic field of $7 \mathrm{~T}$ in amorphous carbon thin films synthesized by chemical vapor deposition. No tendency of saturation of MR is observed up to the magnetic field of 7 T. Shift of structural morphology from disordered amorphous carbon to ordered graphitic like carbon was studied by using Raman, X-ray Diffraction, High Resolution Transmission Electron Microscopy and X-ray Photoelectron Spectroscopy. Negative MR effect increases with the increase in structural order. The mechanism of this negative MR could be ascribed to the Weak Localization and Grain Boundary Scattering Conduction depending on the different temperature regimes.
\end{abstract}

\section{Key words:}

Magnetoresistance; Amorphous Carbon; Transport properties; Weak localization; Grain boundary scattering

\section{Introduction}

Different types of amorphous carbon thin films have been considered for applications to the optical and electronics industry [1, 2]. Among these applications, spintronics application such as magnetoresistance is quite fascinating [3, 4]. 
The allotropes of carbon can comprise different bonding configurations depending upon the hybridization of the four valance electron orbitals. The four-fold symmetry $\mathrm{C}\left(s p^{3}\right)$ hybridization, like in diamond and three-fold symmetry $\mathrm{C}\left(s p^{2}\right)$ hybridization, like in graphite are two major allotrope $[5,6]$. Amorphous carbon (aCarbon) films contain both $s p^{2}$ and $s p^{3}$ hybridized carbon atoms with variable proportions. The proportion of $s p^{2}$ to $s p^{3}$ strongly depends upon the growth conditions [7-9]. In a-Carbon, disorder is a dominant factor to influence the temperature dependence of the conductivity [10-12]. A continuous transition from extended state to localized state is possible by tuning the disorder $[13,14]$.

A lot of reports studied intensively on the structural and transport properties of $\mathrm{Fe}$, Co and Ni doped a-Carbon thin films [15-17], while for pure a-Carbon systems these properties still needed to investigate for further studies. It is important to study the transport properties of these a-Carbon films because it could help to reveal the influence of amorphous nature of such materials on their magnetotransport properties $[18,19]$. Several mechanisms like weak localization [20], grain boundary scattering [21], electron-electron interaction [22] and wave function shrinkage [23], etc. were proposed to understand the transport properties of a-carbon. Even though, these theories are unable to give a good explanation of the origin of transport properties of a-carbon. Thus, further work is necessary to understand the origin of MR for acarbon.

In this work, different disordered a-carbon thin films were fabricated by using chemical vapor deposition (CVD) technique. Structural transition of disordered amorphous to ordered graphitic crystalline structure and its effect on MR was studied. The MR mechanisms were investigated over different temperature regimes.

\section{Material and Methods}

Silicon wafer with a layer of $\mathrm{SiO}_{2}$ of $300 \mathrm{~nm}$ in thickness on the surface were used as substrate to grow the a-carbon thin films. Sonication was performed on the substrates for 30 minutes to remove surface contamination. The alumina tube furnace was evacuated up to $\sim 10^{-1}$ torr. The synthesis of these a-Carbon films was done in three steps. In the first step, the temperature of the tube furnace was raised up to 473 $\mathrm{K}$ with a ramp rate of $5 \mathrm{~K} / \mathrm{min}$. Argon $(\mathrm{Ar})$ and Hydrogen $\left(\mathrm{H}_{2}\right)$ were used as precursor 
gases with flow rates of $160 \mathrm{sccm}$ and $40 \mathrm{sccm}$, respectively. In the second step, the temperature was increased from $473 \mathrm{~K}$ to $1373 \mathrm{~K}$ with the rate of $10 \mathrm{~K} / \mathrm{min}$ and the substrate was annealed at $1373 \mathrm{~K}$ for $15 \mathrm{~min}$. Ethylene $\left(\mathrm{C}_{2} \mathrm{H}_{4}\right)$ was introduced as a carbon source with the flow rate of $40 \mathrm{sccm}$. The growth time was varied from 10 to 30 minutes for producing different ordered structure thin films. The a-carbon films fabricated for 10, 20 and 30 minutes were named as sample S1, S2 and S3, respectively. In the third step, the tube furnace temperature was hold at $1273 \mathrm{~K}$ for 30 minutes. After that, the tube furnace was cooled down to room temperature in the presence of argon and hydrogen.

Raman spectrum was measured by Renishaw InVia Raman microscope using excitation wavelength of $514 \mathrm{~nm}$. High Resolution Transmission Electron Microscopy (HRTEM) (JOEL-2011) was used to investigate the structural morphology of acarbon thin films. X-ray Diffraction was used to study the structural property. Moreover, physical property measurement system (PPMS, Quantum design) was used to investigate the magneto-transport properties. The metal electrodes were prepared by standard indium pressing method. The resistance measurement was done by standard four-probe method.

\section{Results and Discussion}

\section{$3.1 \quad$ Structural properties}

HRTEM was used to investigate the structure of a-Carbon specimens. Amorphous structure was observed for samples S1 and S2 while sample S3 shows polycrystalline structure (Figure1). The lattice d-spacing of crystals in specimen S3 were estimated $\sim 0.21 \mathrm{~nm}$ and $\sim 0.12 \mathrm{~nm}$ corresponding to the (100) and (110) plans (figure 1). The films contain short-range ordered structure and the average grain size of crystallite for sample S3 was estimated $\sim 3 \mathrm{~nm}$. The structural transition was observed from amorphous to polycrystalline carbon from sample $\mathrm{S} 1$ to $\mathrm{S} 3$ as shown by diffraction patterns (inset of figure 1). The sample S3 have more ordered structure as compared to S1 and S2 because sample S3 have much better oriented graphitic structure. 

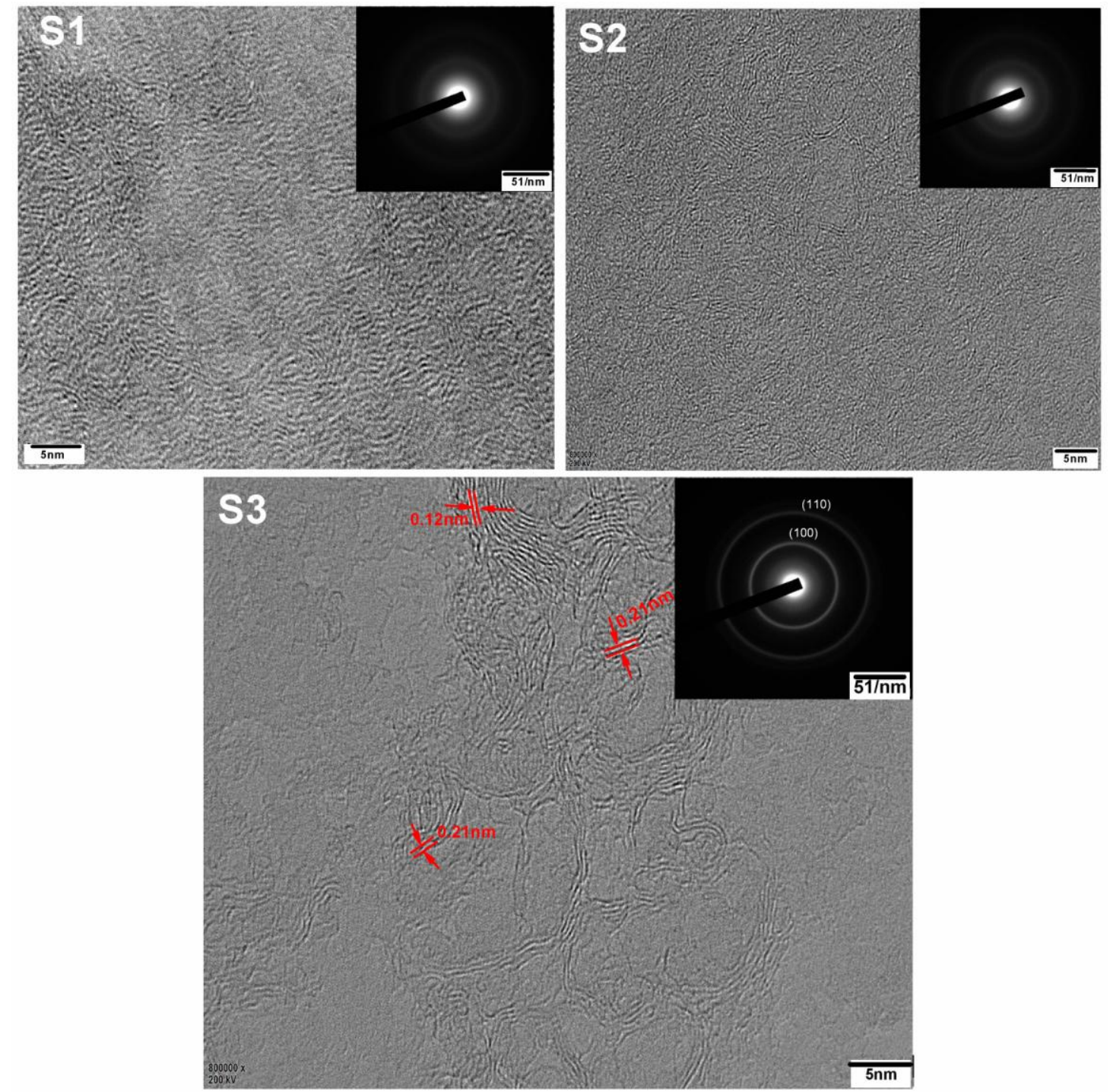

Figure 1. HRTEM image and diffraction pattern for samples S1, S2 and S3.

Atomic Force Microscopy (AFM) was used to estimate the thickness of samples and the thickness was found to be $\sim 20 \mathrm{~nm}, \sim 44 \mathrm{~nm}$ and $\sim 78 \mathrm{~nm}$ for samples $\mathrm{S} 1, \mathrm{~S} 2$ and S3, respectively. Raman spectra of different ordered structured samples (S1 to S3) are shown in figure 2(a). The peak shift and change in Full Width at Half Maximum (FWHM) of the two major peaks corresponding to the D-band and G-band was studied. The peak shifts of D-band and G-band are observed from $\sim 1331 \mathrm{~cm}^{-1}$ to $\sim 1349 \mathrm{~cm}^{-1}$ and $\sim 1576 \mathrm{~cm}^{-1}$ to $\sim 1595 \mathrm{~cm}^{-1}$, respectively, for samples $\mathrm{S} 1$ to $\mathrm{S} 3$ as shown in figure 2(b). The FWHM of D-band decreased from $\sim 122 \mathrm{~cm}^{-1}$ to $\sim 97 \mathrm{~cm}^{-1}$ and the width of the G-band increased from $\sim 69 \mathrm{~cm}^{-1}$ to $\sim 73 \mathrm{~cm}^{-1}$ for sample S1 to S3 are shown in figure 2(c). The D-band is the main focus of interest, as it corresponds to the breathing mode of $A_{l g}$ symmetry in six atoms ring of carbon, which depends on 
the defects to activate. The D-band is more dominant in sample S1 with the maximum FWHM value of $\sim 122 \mathrm{~cm}^{-1}$, which corresponds to the most disordered structure specimen. The decrease in FWHM value of D peak indicates the decrease in structural disorder and the increase in FWHM of G peak signifies the more graphitic like structure. Where as, for sample S3 with the FWHM value of $\sim 97 \mathrm{~cm}^{-1}$ has lowest degree of disorder. So the disorder decreases with the decrease in FWHM value from $\sim 122 \mathrm{~cm}^{-1}$ to $\sim 97 \mathrm{~cm}^{-1}$ from sample $\mathrm{S} 1$ to $\mathrm{S} 3$ as shown in figure 2(c). Furthermore, the decrease in the value of $\mathrm{I}_{\mathrm{D}} / \mathrm{I}_{\mathrm{G}}$ ratio from 2.1 to 1.3 can also be used to estimate the transition of disordered to ordered structure from sample S1 to S3. Thus, the shift of D-band peak position and decrease in FWHM value of D-band may be used for estimation of structural changes for amorphous carbon materials system.
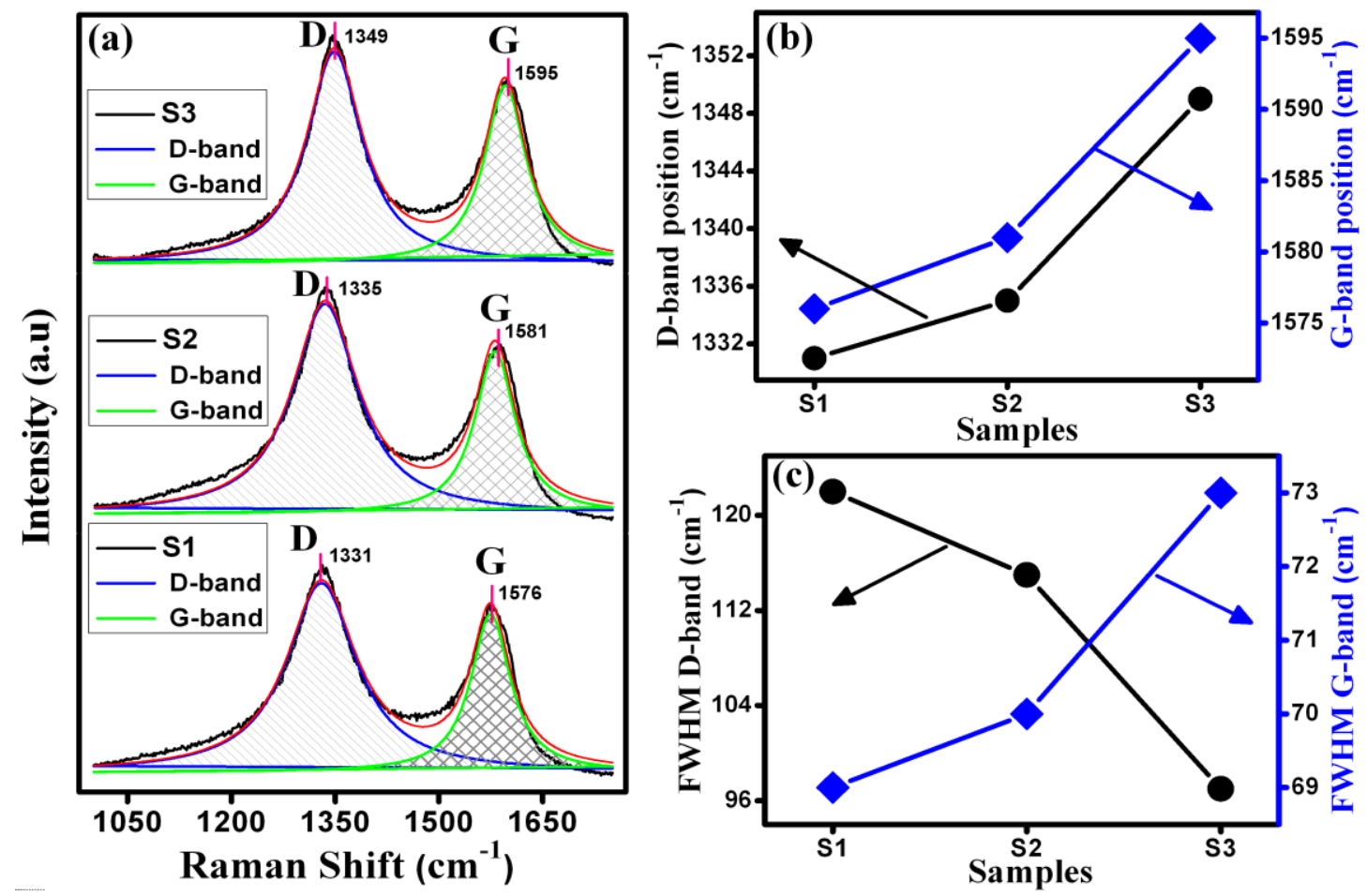

Figure 2. (a) Raman spectra; (b) Peak shift of D-band and G-band position; (c) Change in FWHM of D-band and G-band, for samples S1 to S3.

X-ray Diffraction (XRD) is a powerful tool to study the crystallinity of a material. XRD plots for the samples S1 to S3 are shown in figure 3(a), which indicates the shift of amorphous carbon to hexagonal crystalline graphitic like structure for sample S1 to S3. The sample S1 shows completely amorphous behavior while the sample $\mathrm{S} 2$ have the structural transition trend from amorphous to crystalline structure. The crystalline structure was observed for sample S3 which shows the 
carbon peaks of (002) and (101) planes corresponds to the hexagonal graphitic like carbon at $26^{\circ}$ and $46^{\circ}$, respectively.
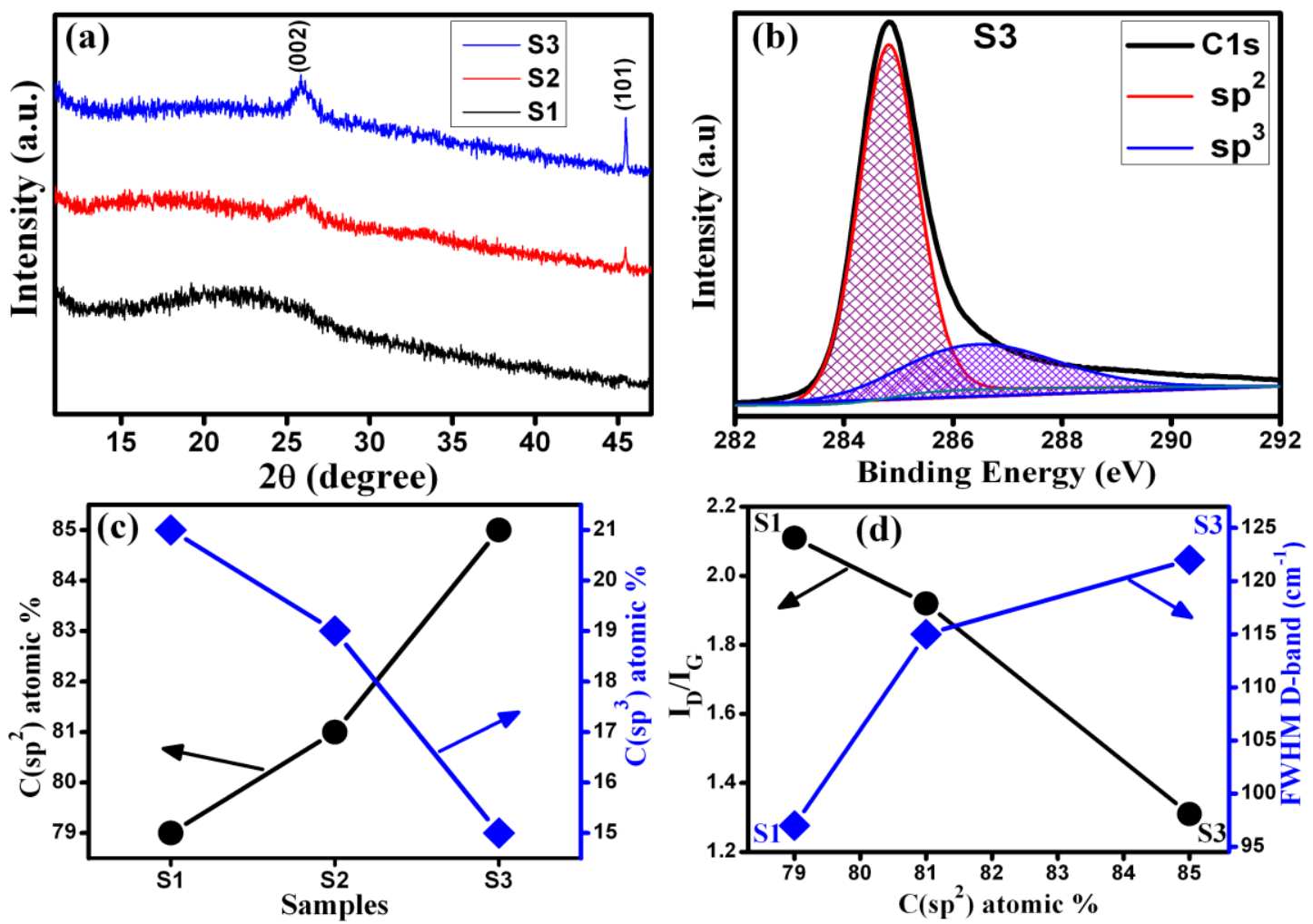

Figure 3. (a) XRD pattern of samples S1 to S3; (b) XPS spectrum of sample S3; (c) $\mathrm{C}\left(s p^{2}\right)$ and $\mathrm{C}\left(s p^{3}\right)$ atomic \%; (d) $\mathrm{I}_{\mathrm{D}} / \mathrm{I}_{\mathrm{G}}$ ratio vs $\mathrm{C}\left(s p^{2}\right)$ atomic \%, for samples $\mathrm{S} 1$ to $\mathrm{S} 3$.

Figure 3(b) shows the XPS spectrum for sample S3 fitted with the lorentzian function to estimate the atomic percentage of $\mathrm{C}\left(s p^{2}\right)$ and $\mathrm{C}\left(s p^{3}\right)$ clusters observed at $\sim 284.6 \mathrm{eV}$ and $\sim 285.9 \mathrm{eV}$, respectively. The atomic percentage of $\mathrm{C}\left(s p^{2}\right)$ in sample $\mathrm{S} 3$ with most ordered structure is $\sim 85 \%$. The atomic percentage of $\mathrm{C}\left(s p^{2}\right)$ is increased from $79 \%$ to $85 \%$ and $\mathrm{C}\left(s p^{3}\right)$ decreased from $21 \%$ to $15 \%$ for samples $\mathrm{S} 1$ to $\mathrm{S} 3$ (figure 3(c)). The atomic percentage ratio of $\mathrm{C}\left(s p^{2}\right) / \mathrm{C}\left(s p^{3}\right)$ is also increased from 3.7 to 5.6 with the increase in structural order from sample S1 to S3.

As the carbon materials having dominant $\mathrm{C}\left(s p^{2}\right)$ clusters are considered as good conductor materials while carbon materials with dominant $\mathrm{C}\left(s p^{3}\right)$ clusters are considered as insulators, the ratio of $\mathrm{C}\left(s p^{2}\right) / \mathrm{C}\left(s p^{3}\right)$ can be used to estimate the better conduction, which is maximum for sample S3. These results also correlate with the resistivity plot as well as with the Raman results (figure $4(b)$ ). The $I_{D} / I_{G}$ ratio which corresponds to the degree of disorder, decreases with the increase of $\mathrm{C}\left(s p^{2}\right)$ atomic 
percentage up to $85 \%$ (figure 3(d)). On the other hand, FWHM of D-band increases with the increase of $\mathrm{C}\left(s p^{2}\right)$ atomic $\%$.

\subsection{Magnetotransport properties}

Figure 4(a) shows that the resistivity decreases with the decrease of structural disorder from $2 \mathrm{~K}$ to $300 \mathrm{~K}$ for all samples. This result is in agreement with the Raman and XPS results. So the decrease of defects and disorder of structure are responsible for better conduction from sample S1 to S3.
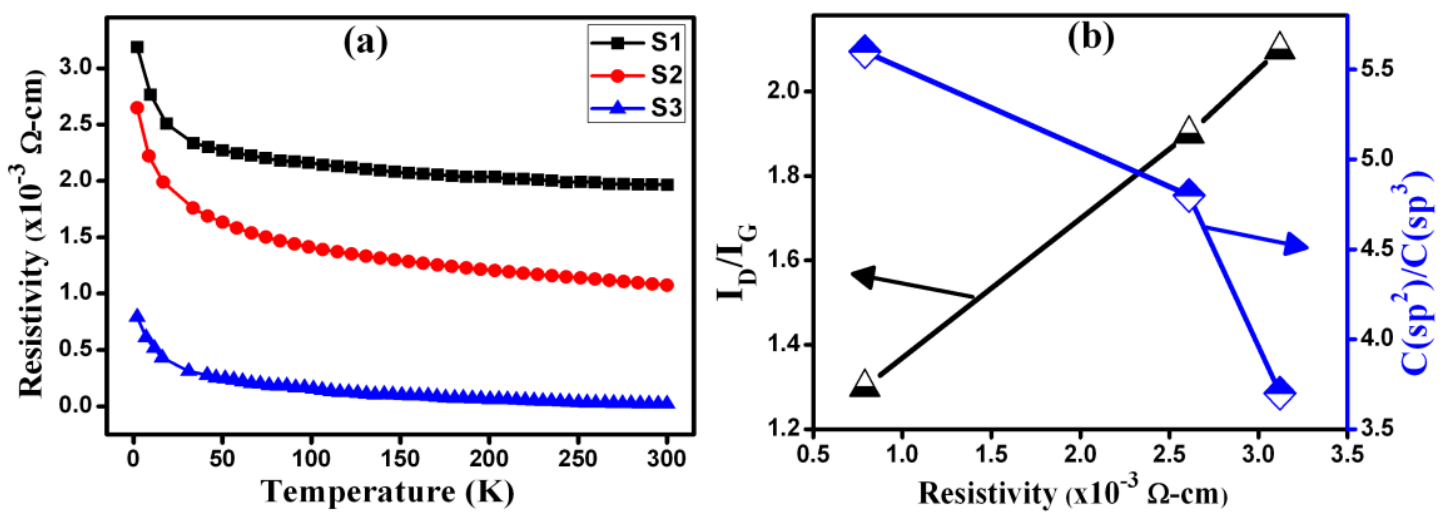

Figure 4. (a) Resistivity as a function of temperature; (b) $\mathrm{I}_{\mathrm{D}} / \mathrm{I}_{\mathrm{G}}$ and $\mathrm{C}\left(s p^{2}\right) / \mathrm{C}\left(s p^{3}\right)$ vs resistivity.

Magnetoresistance is a sensitive local probe for studying the scattering process in disordered amorphous systems and it highly depends on the temperature dependence of conductivity [24]. The MR of sample S1 to S3 increases from $-6 \%$ to $-13 \%$ at $2 \mathrm{~K}$, respectively (figure $5(\mathrm{a})$ ). MR of sample $\mathrm{S} 3$ from $2 \mathrm{~K}$ to $300 \mathrm{~K}$ up to the magnetic field of $7 \mathrm{~T}$ is shown in figure 5(b). The observed negative MR decreases with the increase in temperature from $2 \mathrm{~K}$ to $300 \mathrm{~K}$ and increases with the increase in magnetic field from 1 to $7 \mathrm{~T}$ (figure $5(\mathrm{~b})$ ). MR of $-13 \%$ is observed at $2 \mathrm{~K}$ for sample S3, which has the most ordered structure with lowest resistivity. 

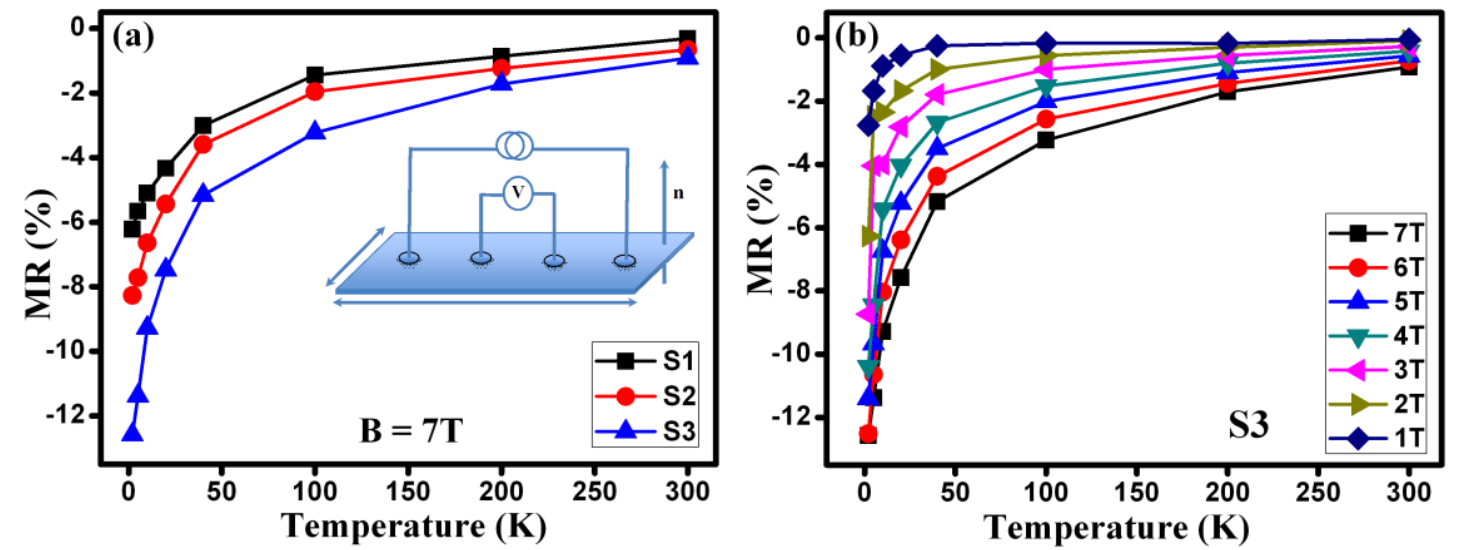

Figure 5. MR as a function of temperature (a) for samples $S 1$ to $S 3$ at $B=7 \mathrm{~T}$; (b) for sample S3 at different magnetic fields.

Figure 6 shows the MR-B curves of samples S1 and S3, from $2 \mathrm{~K}$ to $300 \mathrm{~K}$ in the magnetic field range of $-7 \mathrm{~T}$ to $7 \mathrm{~T}$. The negative MR value decreases with the increase in temperatures. The shape of MR-B curve is changed from cone like shape to parabolic like shape with the increase in temperature from $2 \mathrm{~K}$ to $300 \mathrm{~K}$. The observed shape for transition temperature from cone like to parabolic like MR-B curve for sample $\mathrm{S} 1$ is $\sim 20 \mathrm{~K}$ and for $\mathrm{S} 3$ is $\sim 50 \mathrm{~K}$, respectively. The shape transition temperature shifts to higher temperature with the increase in structural order from S1 to S3. The shape change of MR-B curve may correspond to the difference in MR mechanism at different temperatures.
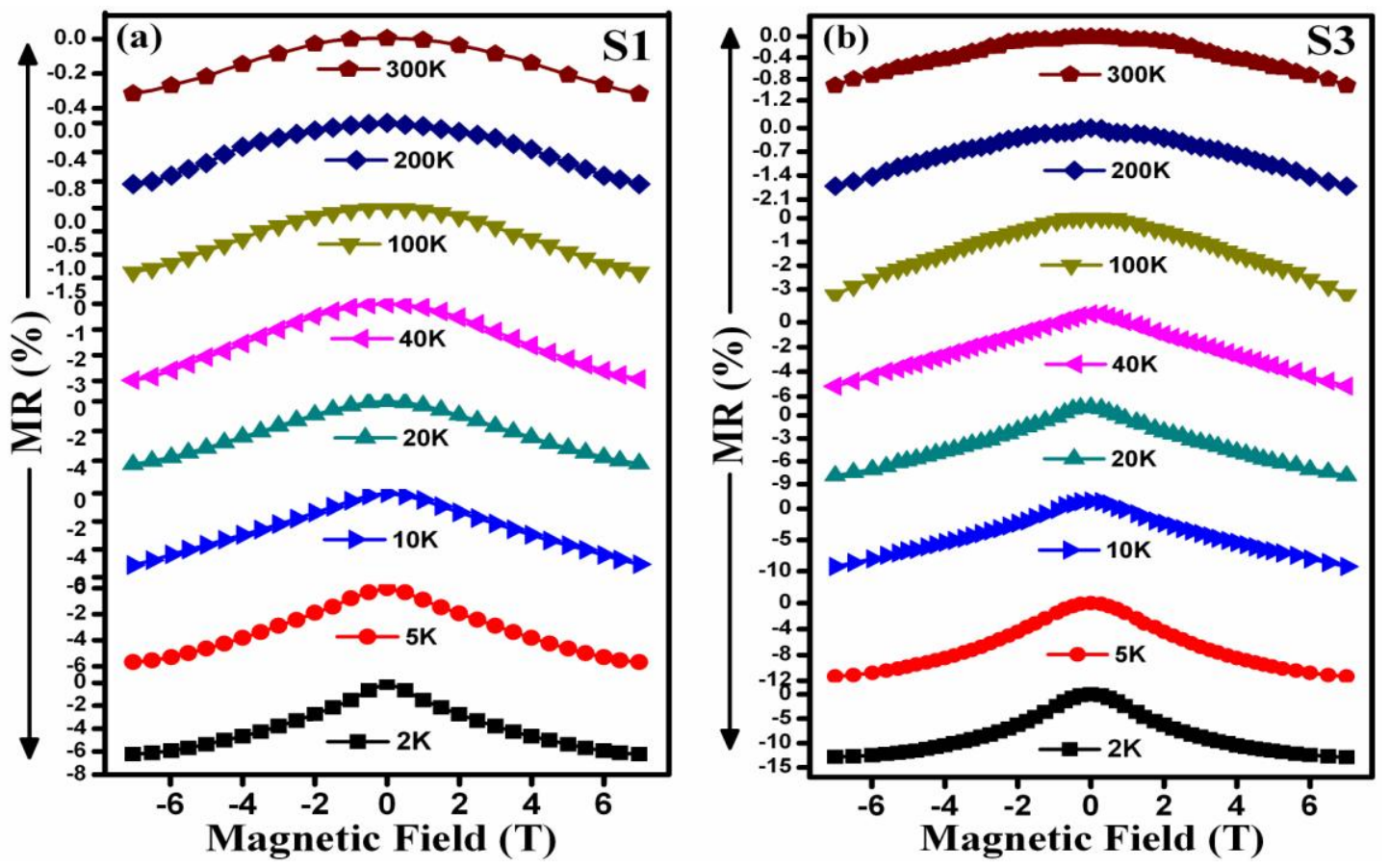

Figure 6. MR-B curve at different temperatures, (a) for sample S1; (b) for sample S3. 
The negative MR in disordered systems could be originated from a variety of mechanisms. Among these mechanisms, weak localization theory (WLT) is a possible mechanism for negative MR. However, negative MR originated from WLT is usually a low temperature $(<100 \mathrm{~K})$ effect, because the inelastic scattering process due to electron-phonon or electron-electron interaction makes the phase incoherence between carrier waves and this effect diminishes in high temperatures [25, 26]. It is also observed that the weak localization effect is dominant up to $\sim 50 \mathrm{~K}$ in sample $\mathrm{S} 3$ (figure 7(a)). Temperature dependent conductivity for WLT is given by [10].

$$
\sigma_{3 D}(T)=\sigma_{o}+\frac{e^{2}}{\hbar \pi^{2}} \frac{1}{a} T^{p / 2}
$$

where $\sigma_{3 D}$ is the conductivity, $\sigma_{o}$ is the Boltzmann transport conductivity, $\hbar$ is reduced planks constant, $a \approx K_{F}^{-1}$ is the inverse of Fermi wave number and $p$ is bond fraction constant. The WLT fits well for the R-T curve in temperature ranges of $\sim 2-$ $20 \mathrm{~K}, \sim 2-30 \mathrm{~K}$ and $\sim 2-50 \mathrm{~K}$ for samples S1, S2 and S3 respectively (Figure 7(a)). So the WLT dominancy also shifts to the higher temperature with the increase in structural order from sample S1 to S3.
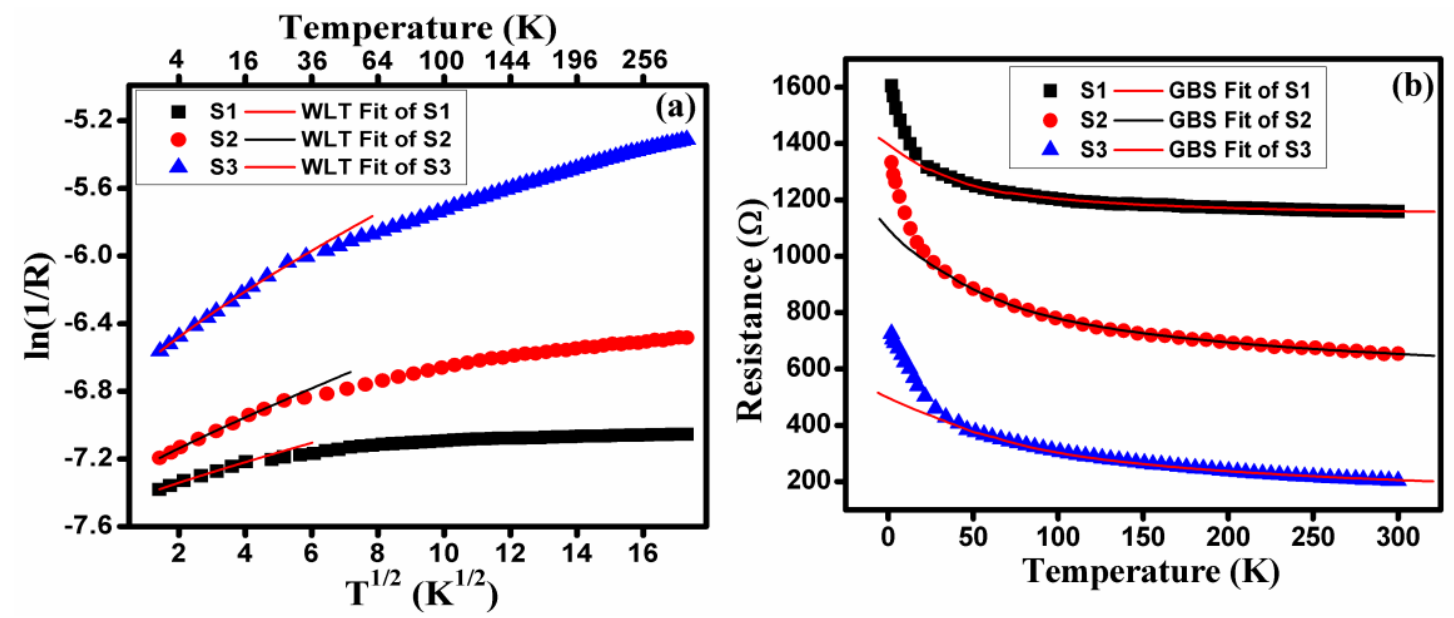

Figure 7. R-T curve fit for temperatures $2 \mathrm{~K}$ to $300 \mathrm{~K}$ for all samples (a) Weak localization theory; (b) GBS conduction model.

The quantum interference of different carrier conduction is involved at low temperature in WLT and the time reversal symmetry can be broken by magnetic field. This effect will decrease the interference so that the resistance of the system will decrease. Moreover, the effect of magnetic field on the weak localization of electrons is similar to the inelastic scattering. It suppresses the phase coherence between the 
partial wave associated with an electron and this reduces the localization effect, which causes the decrease of resistance with increase in magnetic field [26]. It is also observed that the WLT effect is more sensitive to the lower temperatures and decreases rapidly with the increase in temperature for all samples. Thus, the WLT effect is dominant in low temperature regions.

The diffused scattering at crystallite boundaries is another possible mechanism of negative MR in polycrystalline or highly disordered materials [25]. The presence of the crystallites in a-Carbon samples indicates the scattering phenomenon at the grain boundaries, which varies to different temperature range depending upon the grain size of the clusters in different samples. GBS effect is studied at higher temperature regions in order to explain the negative MR. The R-T curves are fitted with GBS conduction model with the equation given by [22].

$$
=A\left(1+b T^{p}\right) C^{D /\left(1+b T^{p}\right)}
$$

where $\rho$ is the resistivity and $p$ is the material dependent constant. $A$ is the probability of carriers to pass through the single grain boundary. GBS model fits well for temperature range of $\sim 20-300 \mathrm{~K}$ for sample S1, while $\sim 30-300 \mathrm{~K}$ for sample S2 and $\sim 50-300 \mathrm{~K}$ for sample S3. This temperature range shifts to the higher temperature with the increase in both grain size of crystals and order of the structure for sample S1 to S3. GBS theory indicates that the charge carrier's bends due to the Lorentz force when the magnetic field is applied normal to the plan of the samples, which will reduce the sample resistance [26]. This decrease in sample resistance will lead to the negative MR effect in high temperature region. Thus, the GBS effect limits to explain the negative MR only in high temperature regions.

As the low temperature regions are more sensitive to the magnetic field, the magnetic field effect is more evident in WLT effect as compared to the magnetic effect in GBS conduction. In GBS effect, the crystals in amorphous carbon matrix system are specifically concerned to the $\mathrm{C}\left(s p^{2}\right)$ clusters, which are also dominant in samples S1 to S3. Therefore, for both GBS and WLT, the C $\left(s p^{2}\right)$ clusters are more responsible for the negative MR phenomenon. 


\section{Conclusions}

Semiconducting a-carbon thin films of different ordered structure were fabricated by CVD technique. The evolution of nano-graphitic like crystalline phase in the a-Carbon was witnessed. A novel way to control the degree of structural disorder of amorphous carbon by CVD method was proposed. Evidence for the increase in ordered structure and increase in $\mathrm{C}\left(s p^{2}\right)$ were also observed. In ordered structure specimen negative MR of $13 \%$ was observed at $2 \mathrm{~K}$ under the magnetic field of $7 \mathrm{~T}$. The MR mechanism was investigated by using Grain boundary scattering conduction model and Weak localization theory. The anomalous shape change of MR-B curve was found to associate with the different MR mechanisms. The total MR was considered as the combined effect of GBS induced MR and WLT induced MR.

\section{Acknowledgements}

We would like to acknowledge the financial support by The National Science Foundation of China (Grant Nos. 11234007, 51471093 and 11674190). Moreover, Mr. Saleemi highly acknowledges to the Chinese Scholarship Council for financial assistance during my PhD.

\section{References}

[1] Caihua Wan, Xiaozhong Zhang, Xili Gao, Jimin Wang, Xinyu Tan, Geometrical enhancement of low-field magnetoresistance in silicon, Nature, 477 (2011) 304.

[2] A.H. R. Xu, T. F. Rosenbaum, M. L. Saboungi, J. E. Enderby, P. B. Littlewood, Large magnetoresistance in non-magnetic silver chalcogenides, Nature, 390 (1997) 57.

[3] T.T. S. A. Solin, D. R. Hines, J. J. Heremans,, Enhanced Room-Temperature Geometric Magnetoresistance in Inhomogeneous Narrow-Gap Semiconductors, Science, 289 (2000) 1530.

[4] A.S. Saleemi, R.U.R. Sagar, R. Singh, Z. Luo, X. Zhang, Angle dependent magnetotransport in transfer-free amorphous carbon thin films, J. Phys. D: Appl. Phys, 49 (2016) 415005. 
[5] Y.C. Jiang, Z.P. Wu, W. Bao, S.J. Xu, J. Gao, Tunable positive magnetoresistance effect of Co-doped amorphous carbon films, J. Appl. Phys, 111 (2012) 07C510.

[6] S.F. M. Fath, A. A. Menovsky, Y. Tomioka, J. Aarts, J. A. Mydosh, Spatially Inhomogeneous Metal-Insulator Transition in Doped Manganites, Science, 285 (1999) 1540.

[7] G. Bergmann, Magnetic Screening of Fe Impurities in Mg, Phys. Rev. Lett, 57 (1986) 1460.

[8] L. Kumari, S.V. Subramanyam, S. Eto, K. Takai, T. Enoki, Metal-insulator transition in iodinated amorphous conducting carbon films, Carbon, 42 (2004) 2133.

[9] J. Panda, S.N. Saha, T.K. Nath, Room temperature giant positive junction magnetoresistance of $\mathrm{NiFe}_{2} \mathrm{O}_{4} / \mathrm{n}-\mathrm{Si}$ heterojunction for spintronics application, Physica B: Cond. Matt, 448 (2014) 184.

[10]R.U.R. Sagar., X. Zhang., J. Wang., Chengyue Xiong, Negative magnetoresistance in undoped semiconducting amorphous carbon films, J. Appl. Phys, 115 (2014) 123708.

[11]Z.W. Fan, P. Li, L.T. Zhang, W.B. Mi, E.Y. Jiang, H.L. Bai, Electrical transport properties and room-temperature positive magnetoresistance of $\mathrm{Fe}_{3} \mathrm{O}_{4} / \mathrm{a}-\mathrm{C} / \mathrm{n}-\mathrm{Si}$ junctions, Thin Solid Films, 520 (2012) 3641.

[12]X. Gao, X. Zhang, C. Wan, J. Wang, X. Tan, D. Zeng, Temperature-dependent resistive switching of amorphous carbon/silicon heterojunctions, Diamond Rel. Mat, 22 (2012) 37.

[13] V. Bayot, L. Piraux, J.P. Michenaud, J.P. Issi, M. Lelaurain, A. Moore, Twodimensional weak localization in partially graphitic carbons, Physical Rev. B, 41 (1990) 11770.

[14] J. Wang, X. Zhang, C. Wan, J. Vanacken, V.V. Moshchalkov, Magnetotransport properties of undoped amorphous carbon films, Carbon, 59 (2013) 278-282.

[15] P. Tian, X. Zhang, Q.Z. Xue, Enhanced room-temperature positive magnetoresistance of a-C:Fe film, Carbon, 45 (2007) 1764.

[16]D.D. Zhu, Anomalous positive magnetoresistance in $\mathrm{Co}_{\mathrm{x}}-\mathrm{C}_{1-\mathrm{x}}$ granular films on Si substrates, J. Appl. Phys, 95 (2004) 1906. 
[17]X.C. Wang, W.B. Mi, E.Y. Jiang, H.L. Bai, Large magnetoresistance observed in facing-target sputtered $\mathrm{Ni}$-doped $\mathrm{CNx}$ amorphous composite films, Acta Materialia, 55 (2007) 3547.

[18]A. Grill, Electrical and optical properties of diamond-like carbon, Thin Solid Films, 355-356 (1999) 189.

[19]J.D. Carey, S.R.P. Silva, Disorder, Clustering and Localization Effects in Amorphous Carbon, Physical Rev. B, 70 (2004) 235417.

[20]Q.Z. Xue, X. Zhang, Anomalous electrical transport properties of amorphous carbon films on Si substrates, Carbon, 43 (2005) 760.

[21] A.C. Ferrari, D.M. Basko, Raman spectroscopy as a versatile tool for studying the properties of graphene, Nat. Nano, 8 (2013) 235.

[22]R.U.R. Sagar, A.S. Saleemi, X. Zhang, Angular magnetoresistance in semiconducting undoped amorphous carbon thin films, J. Appl. Phys, 117 (2015) 174503.

[23] C. K. Chung, A. Nautiyal, T. S. Chen, Y.L. Chang, Grain boundary scattering for temperature coefficient of resistance (TCR) behaviour of $\mathrm{Ta}-\mathrm{Si}-\mathrm{N}$ thin films, $\mathrm{J}$. Phys. D: Appl. Phys, (2008) 5.

[24] A. Iwase, N. Ishikawa, T. Iwata, Y. Chimi, Negative magnetoresistance of pyrolytic carbon and effects of low-temperature electron irradiation, Physical Rev. B, 60 (1999) 10811.

[25] Y.B. Zhou, B.-H. Han, Z.-M. Liao, H.-C. Wu, D.-P. Yu, From positive to negative magnetoresistance in graphene with increasing disorder, Applied Phys. Lett. 98 (2011) 222502.

[26] Y. Hishiyama, H. Irumano, Y. Kaburagi, Y. Soneda, Structure, Raman scattering, and transport properties of boron-doped graphite, Physical Rev. B, 63 (2001) 245406. 

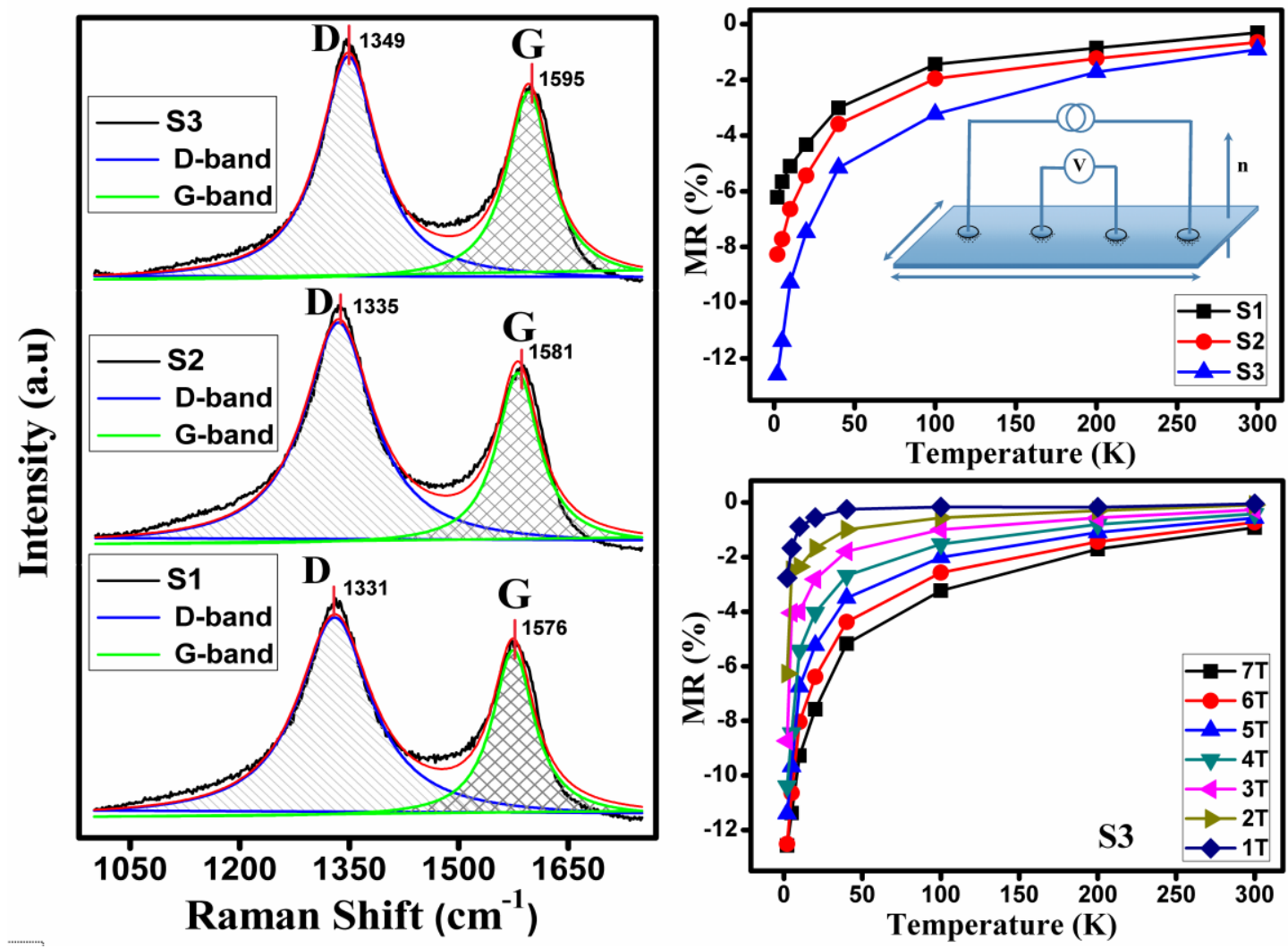